\title{
THE RELATIONSHIP OF ENERGY FLOW AT THE MAGNETOPAUSE TO GEOMAGNETIC ACTIVITY
}

\author{
D. N. Baker and E. C. Stone
}

California Institute of Technology, Pasadena, California, 91125

Abstract. Properties of the energetic electron (E $>200 \mathrm{keV}$ ) magnetopause layer along the distant magnetotail have been studied with Caltech instrumentation aboard IMP-8 for approximately 60 spacecraft orbits. The cross-sectional area of the layer appears to increase by a factor of $\sim 5$ with increasing geomagnetic activity, and the average unfirectional electron flux within the layer increases by a factor of $\sim 4$. The energy carried by electrons $\gg 200 \mathrm{keV}$ ranges from $\sim 10^{14}$ ergs sec $^{-1}$ to $\sim 10^{15}$ ergs $\mathrm{sec}^{-1}$. Extrapolation to include all electrons $>1 \mathrm{keV}$ suggests total energy flow ranging from $\sim 3 \times 10^{15}$ ergs $\mathrm{sec}^{-1}$ at quiet times to

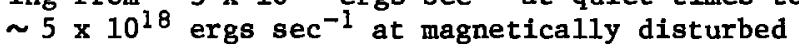
times.

\section{Introduction}

A layer of energetic electrons lying primarily outside the magnetopause is found over most of the magnetospheric surface. This layer had previously been studied extensively at high latitudes near the dusk-dawn meridional plane (Meng and Anderson, 1970, 1975; Domingo et al., 1974, 1977). Recently, Baker and Stone (1977b) have shown both that the energetic electron magnetopause layer is persistently present along the distant magnetotall at essentially all latitudes and that the $₹ 200 \mathrm{keV}$ electrons within the layer are strongly streaming tailward along the local magnetic field. A substantial tailward energy transport in the energized plasma was inferred.

In the present study we have utilized data from 101 magnetopause layer (MPL) crossings and have investigated the dependence of the MPL properties on geomagnetic activity using electrons $(E \geqslant 200$ $\mathrm{keV}$ ) as measured with the wide-geometry detection mode of the Caltech Electron/Isotope Spectrometer (EIS) aboard IMP-8 (see Baker and Stone (1977a) for Instrumental deta1ls). We also extrapolate our observations to much lower electron energies and discuss the implied energy dissipation required to maintain such energy flow.

Magnetopause crossings are identified using plasma analyzer data (L.A. Frank, private communication) and magnetic field data (R.P. Lepping and N.F. Ness, private communication) obtained concurrently with other IMP-8 instrumentation.

\section{Observations}

In Figure 1, we show an example of a typical IMP-8 pass through the northern portion of the tail. In the upper part of Figure 1 are the $X-Y$ and $Y-Z$ plane projections of the spacecraft trajectory in the geocentric solar magnetospheric (SM) coordinate system, w1th dots at 12-hour intervals along the path.

The horizontal shaded bar in the central portion of Figure 1 delineates the various plasma

Copyright 1977 by the American Geophysical Union. regions in which the spacecraft was located at any particular time. Magnetosheath conditions are shown by the cross-hatched shading. Plasma 'boundary layer' regions are shown by the dashed horizontal lines. These regions show substantial bulk fon flow, but the plasmas have lower temperatures and densities than observed in the magnetosheath. The plasma-void high-tail lobes of the magnetotall appear as unshaded gaps, whereas regions of ambiguous flow are shown with dotted shading.

The bottom panel of Figure 1 shows the time profile of the intensity of energetic electrons for this period. Interplanetary fluxes were at their normal level when an abrupt encounter with the MPL was seen at $\sim 2000$ UT of day 153 . The intensity enhancement lasted until the magnetopause was crossed at $\sim 1900$ UT of day 154 . The electrons were seen to be streaming strongly tail-
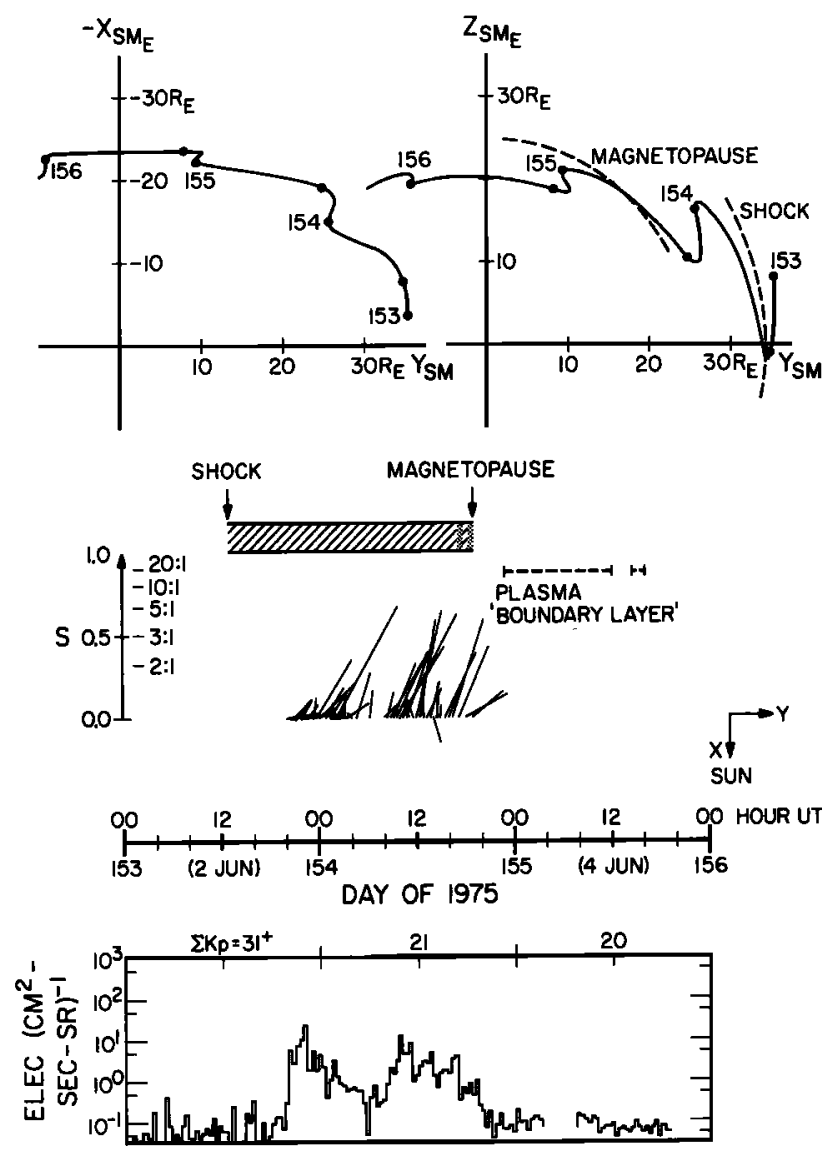

F1gure 1. IMP-8 trajectory information (upper portion of the figure), the observed flux timeprofile of electrons with $\mathrm{E} \geqslant 200 \mathrm{keV}$ (lowest pane1), and observed magnetosheath plasma conditions (shaded horizontal bar). The streaming of $\geqslant 200$ $\mathrm{keV}$ electrons is shown by the plotted anisotropy vectors in the central part of the figure. 


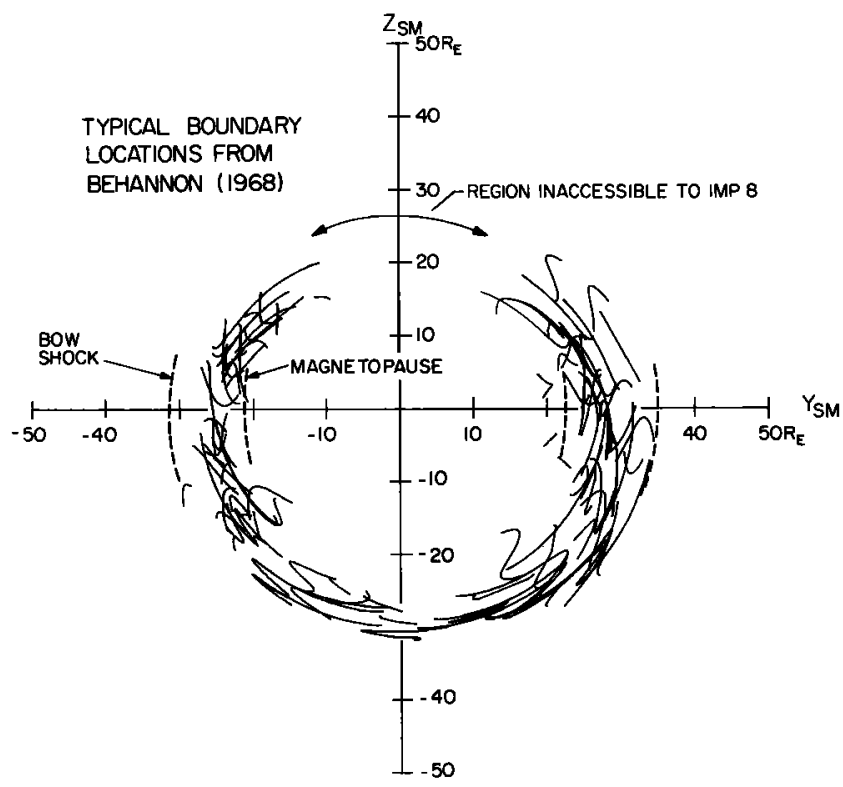

Figure 2. The 101 IMP-8 magnetopause layer crossings used in the present study. The $\mathrm{Y}-\mathrm{Z}$ plan projection of the spacecraft trajectory has been made visible for times during which enhanced intensities and flows of the layer were observed.

ward within the layer. Inside the magnetopause, intensities were at background levels. Moderate to disturbed conditions are indicated by the values of $\Sigma \mathrm{Kp}$ in the lower portion of the figure.

In Figure 2 we show the 101 magnetopause layer crossings obtained with the IMP-8 EIS during 1973, 1974, and 1975 which are used in this study. The spacecraft trajectory has been made visible for times during which enhanced intensities and energetic electron flows of the MPL were observed.

Certain asymmetries present in Figure 2 are artifacts of the IMP- 8 orbit. The orfentation of the major axis of the spacecraft orbit was such that coverage extends all the way around the southern magnetopause surface. As indicated in the figure, TMP-8 did not reach adequately high northern latitudes to completely sample the northern MPL, but it seems reasonable to assume a completely annular cross-section. The average (or typical) magnetopause and bow shock locations found by Behannon (1968) are sketched in for reference in the flgure. Although these are useful guides, it is to be emphasized that in most cases the inner edge of the MPL is the magnetopause identified by local plasma and/or magnetic field data.

An estimate of the average energy flow $\langle P\rangle$ down the layer is given by

$$
\langle\mathrm{P}\rangle \sim \mathrm{A}_{\mathrm{L}} \cdot\langle\mathrm{J}\rangle \cdot\langle\mathrm{E}\rangle \quad(\mathrm{E} \geqslant 200 \mathrm{keV})
$$

where $A$ is the cross-sectional area of the annular layer and $\langle E\rangle$ is the average energy per electron. $A_{L}$ is approximately given by:

$$
A_{L} \sim 2 \pi \rho_{1} \cdot \Delta \rho
$$

where $\rho_{1}$ is the distance to the Inner edge of the MPL and ${ }^{1} \Delta \rho$ is the layer thickness. Since the tailward streaming within the MPL ordinarily produces a highly peaked angular distribution when viewed with the elght $45^{\circ}$ sectors of the EIS (cf. Figure
2 of Baker and Stone $(1977 \mathrm{~b}))$, we estimate $\langle\mathrm{J}\rangle$ as:

$$
\langle\mathrm{J}\rangle=\left\langle\mathrm{c}_{\mathrm{p}}\right\rangle / \mathrm{g \tau} \quad(\mathrm{E} \geqslant 200 \mathrm{keV}) .
$$

Here $\langle\mathrm{J}\rangle$ is the directional flux through $A$ and $\left\langle C_{p}\right\rangle$ is the number of counts per sample in the peak sector, both averaged over the MPL encounter period. The parameter $g$ is the unidirectional geometric factor for electrons $\left(\sim 2.6 \mathrm{~cm}^{2}\right)$ and $\tau$ is the accumulation time per sample in the peak sector $(6.8 \mathrm{sec}$ for the 4.1-minute averages used here).

We find that each component of Eq. (1) varies from one MPL crossing to the next and is correlated with geomagnetic activity as measured by Kp. In Figure 3 we show the apparent magnetopause layer thicknesses plotted versus the daily sum of $\mathrm{Kp}$ ( $\Sigma \mathrm{Kp}$ )approprlate to the particular layer crossing. An easily discernible trend is present, and the least-squares fit to the data (shown as the dashed line) is:

$$
\Delta p=(0.78 \pm 0.25)+(0.10 \pm 0.01) \cdot \Sigma \mathrm{Kp} .
$$

Hence, the value of $\Delta \rho$ ranges from $\sim 1 R_{E}$ at very low values of $\Sigma \mathrm{K}_{\mathrm{p}}$ to $\sim 5 \mathrm{R}_{\mathrm{E}}$ at very disturbed times.

We use the phrase "apparent layer thickness" because our analysis assumes stationary boundaries (e.g., a stationary magnetopause surface). The scatter of the points in Figure 3 (typically $\pm 1 R_{E}$ ) may be due to magnetopause motions during the observation.

We also find that $\left\langle C_{p}\right\rangle$ in Eq. (3) above may be represented by:

$$
\left\langle\mathrm{c}_{\mathrm{p}}\right\rangle=(16 \pm 3) \cdot \exp [\Sigma \mathrm{Kp} \cdot(0.044 \pm 0.007)] \text {. }
$$

to within a factor of $\sim 2$, except for MPL crossings within $\pm 10^{\circ}$ of the $\mathrm{Z}$ side. Average intensities In these low-latitude dawn crossings are higher than for other crossings at a given $\Sigma \mathrm{Kp}$, typically by a factor of 2 to 5 .

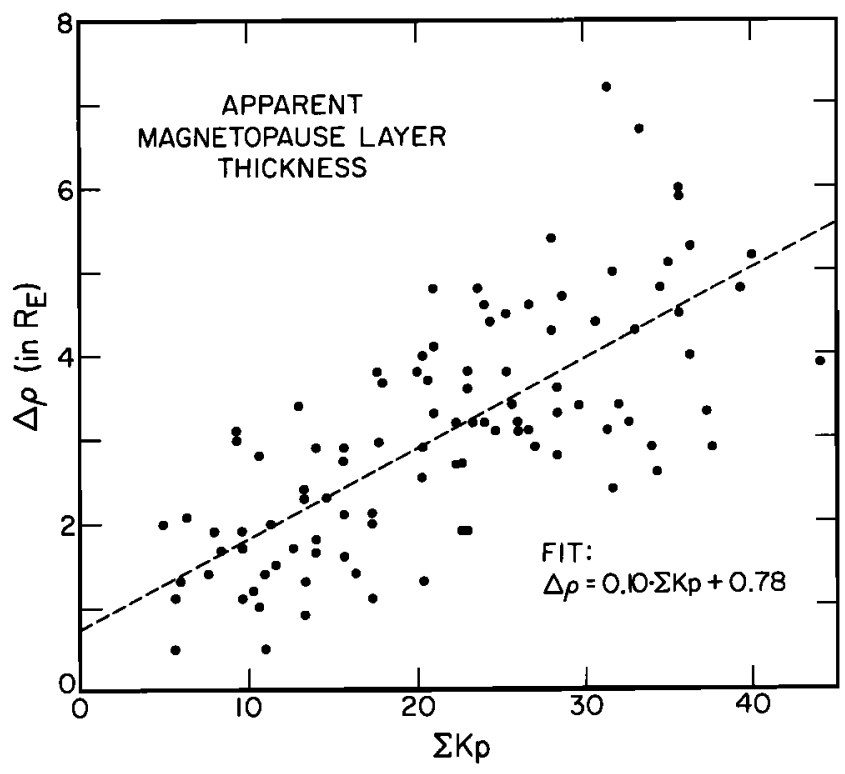

Figure 3. The apparent MPL thicknesses versus the daily sum of $\mathrm{Kp}$ for the layer crossing. A least-squares fit is shown. 
The low-latitude dawn values of $\left\langle c_{p}\right\rangle$, we feel, are localized "hot spots" which may be near a source region for the rest of the layer and as such do not typify the entire MPL at the time of those crossings.

We find a mild, but significant, ¿Kp-dependence of the spectral index $(\gamma)$ for the power law differential electron energy spectra within the MPL. A fit to the data gives:

$$
\gamma=(2.8 \pm 0.1)+(0.020 \pm 0.004) \cdot \Sigma \mathrm{Kp} .
$$

Hence, $\gamma$, as averaged over the layer, increases from $\leqslant 3$ to $\sim 3.5$ with increasing magnetic activity.

Using the average values of $A,\langle J\rangle$, and $\langle E\rangle$ determined in each MPL crossing we have computed the average energy flows shown in Figure 4 for electrons with $E \geqslant 200 \mathrm{keV}$. The energy flow in the MPL increases with increasing $\Sigma K p$ up to a typical average value of $\sim 10^{15} \mathrm{ergs} \mathrm{sec}^{-1}$ during disturbed times. The formally computed energy flows for the low-latitude dawn points are shown in the figure as open circles and are often seen to correspond to abnormally large flows. The least-squares fit to the solid dots in Figure 4 (shown as the dashed line) gives:

$$
\begin{aligned}
\langle\mathrm{P}\rangle\rangle & 200 \mathrm{keV}=(5.2 \pm 0.1) \\
& \quad \times 10^{13} \cdot \exp [\Sigma \mathrm{Kp} \cdot(0.075 \pm 0.007)] .
\end{aligned}
$$

To within a factor of $\sim 2$, the fit includes 68\% of the computed energy flows at any $\Sigma \mathrm{Kp}$.

\section{Discussion and Summary}

The energy flow actually observed with the EIS represents only a portion (possibly a very small portion) of the total energy dissipated at the magnetopause. The published directional electron energy spectra of Reiff and Reasoner (1975) taken at lunar orbit suggest an extrapolation down to

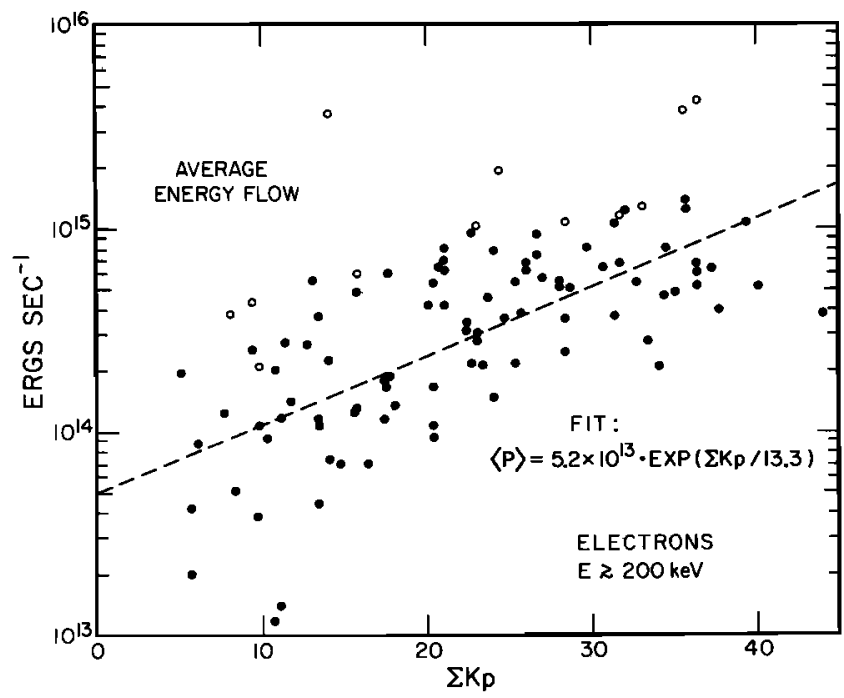

Figure 4. The computed energy flow through the MPL where open circles represent low-latitude dawn crossings of the layer while solid points represent all other crossings. A least-squares fit (to the solid points) is shown.

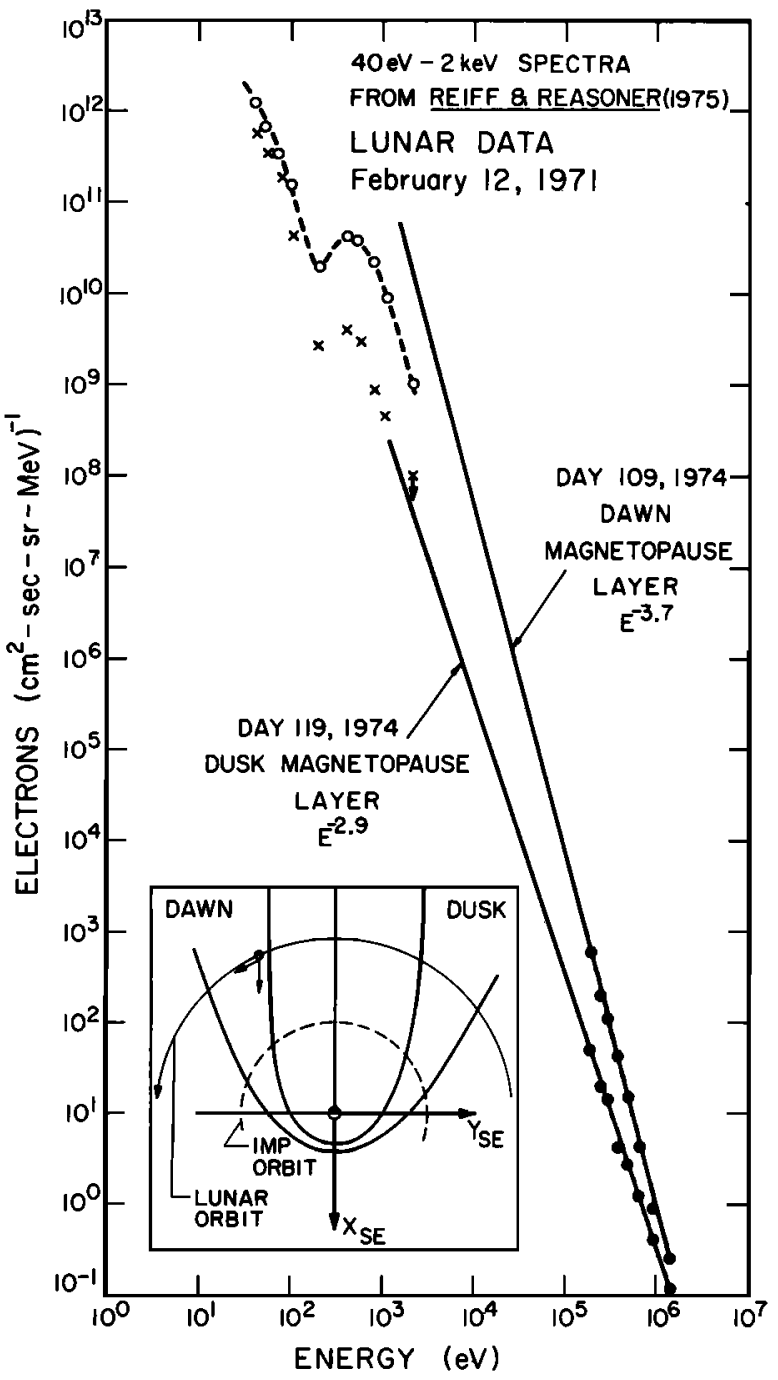

Figure 5. Typical low-energy directional electron spectra measured near the dawn magnetopause (at lunar orbit) compared to the extrapolated differential electron energy spectra typically measured by the IMP-8 EIS within the magnetopause layer.

$E \sim 1 \mathrm{keV}$. In Figure 5 we show a combination of the Reiff and Reasoner data (for electrons between $40 \mathrm{eV}$ and $2 \mathrm{keV}$ ) and two typical limiting cases for the EIS differential electron spectra between $160 \mathrm{keV}$ and $2 \mathrm{MeV}$ extrapolated down to $1 \mathrm{keV}$. The data of Reiff and Reasoner were obtained just outside of the magnetopause (see inset schematic). The open circles represent the spectrum of particles flowing approximately parallel to the magnetopause while the $X^{\prime} s$ represent the spectrum at $60^{\circ}$ to the magnetopause. A relatively isotropic electron component is seen for $40 \mathrm{eV} \leqslant \mathrm{E} \leqslant 200 \mathrm{eV}$, while a highly anisotropic electron component is seen at several hundred $\mathrm{eV}$ and above. Thus, at energies above $200 \mathrm{eV}$ Reiff and Reasoner may have observed the low-energy part of an isotropic electron spectrum similar to that observed with the IMP-8 EIS nearer the earth.

For illustrative purposes, therefore, we carry out an extrapolation of the energy flow, noting that if the observed differential power law ex- 


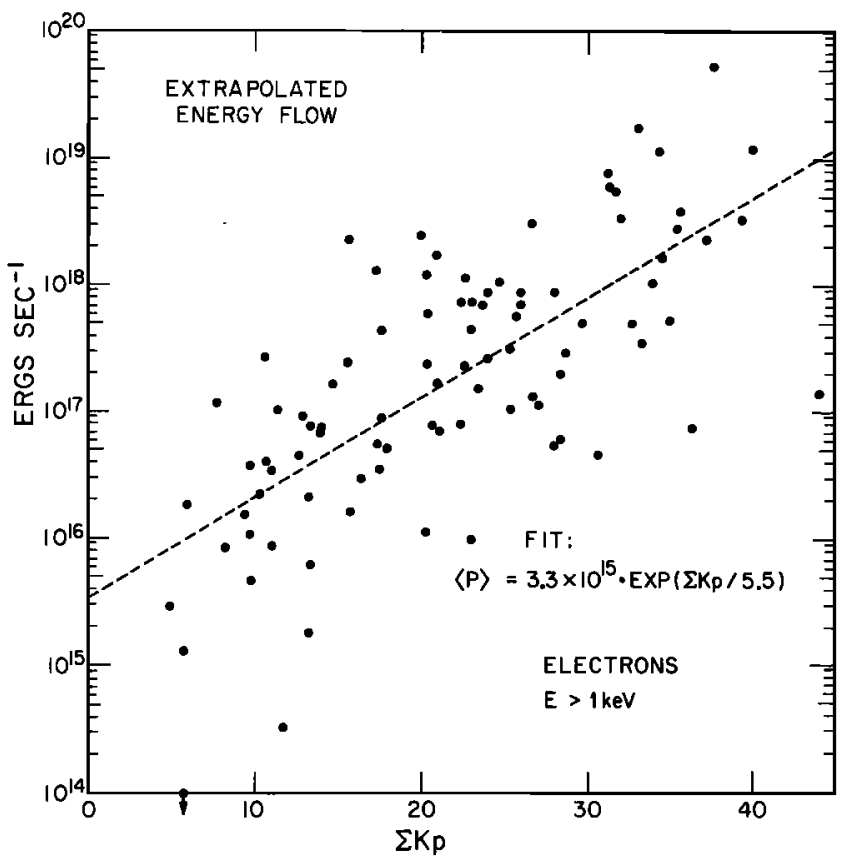

Figure 6. The total energy flow in the MPL inferred to be carried by electrons with $\mathrm{E}>1 \mathrm{keV}$. A least-squares fit is shown.

tended down to $1 \mathrm{keV}$, the integral flux would be larger by a factor of $(200)^{\gamma-1}$ and the average energy would be a factor of 200 smaller, resulting in the relationship

$$
\langle P\rangle>1 \mathrm{keV}^{\sim}\langle P\rangle>200 \mathrm{keV} \cdot(200)^{\gamma-2} \text {. }
$$

Eq. (7) emphasizes that even the relatively weak $\Sigma \mathrm{KP}_{\mathrm{p}}$-dependence of $\gamma$ shown in Eq. (5) will strongly affect the $\Sigma \mathrm{Kp}$-dependence of $\langle P\rangle$.

Figure 6 shows the extrapolated energy flows versus $\Sigma \mathrm{Kp}$ where each point of Figure 4 has been recomputed using Eq. (7). (The low-latitude dawn points have been excluded). The extrapolation suggests a total flow of energized electrons corresponding to $10^{15}-10^{16}$ ergs $\mathrm{sec}^{-1}$ at magnetically quiet times, increasing to $10^{18}-10^{19} \mathrm{ergs} \mathrm{sec}^{-1}$ at disturbed times. The least-squares fit, replicating the data to within approximately an order of magnitude, is given by:

$$
\begin{aligned}
\langle P\rangle\rangle 1 \mathrm{keV} & =(3.3 \pm 0.2) \\
\times 10^{15} \cdot \exp & {[\Sigma \mathrm{Kp} \cdot(0.18 \pm 0.02)] . }
\end{aligned}
$$

Though our extrapolation is to a markedly different particle energy regime than that in which we make our direct observations, several conclusions would seem warranted by our results: (1) At essentially all times and under all geomagnetic conditions, there is apparently substantial energy dissipation at or near the magnetopause; (2) Much of this dissipated energy appears as a highly anisotropic, highly energetic tail on the magnetosheath plasma electron distribution; and (3) The amount of energy flowing along the downstream magnetopause shows a substantial dependence on $\mathrm{Kp}$.

Heikkila (1975) has pointed out that merging at the magnetopause should be accompanied by a gain in the kinetic energy of plasma particles which might appear in a layer near the downstream magnetopause with a corresponding energy flow of up to $\sim 5 \times 10^{18}$ ergs $\mathrm{sec}^{-1}$. Our observations at $E \gg 200 \mathrm{keV}$ suggest a layer structure near the magnetosphere and the extrapolation down to $\sim 1 \mathrm{keV}$ suggests that a major portion of the predicted energy flow may be carried by fast electrons. If direct observations of the entire layer confirm the extrapolation even to within an order of magnitude, such observations would imply that the conversion of magnetic energy into electron kinetic energy is a very efficient process.

The apparent absence of a corresponding 'layer' of energized protons along the downstream magnetopause may also be an important aspect. Recent work has shown that low-frequency turbulence, due to the modified twó-stream instability, can acce1erate electrons (but not protons) to relativistic energies (Lampe and Papadopoulos, 1977). Given the condition that the electron temperature equal or exceed the lon temperature, such stochastic electron acceleration along fleld lines is expected to take place in the wake of collisionless shocks associated with magnetic merging.

Acknowledgments. This work was supported in part by the National Aeronautics and Space Administration under contract NAS5-11066 and Grant NGR 05-002-160.

\section{References}

Baker, D.N., and E.C. Stone, Observations of energetic electrons ( $>200 \mathrm{keV}$ ) in the earth's magnetotafl: Plasma sheet and fireball observations, J. Geophys. Res., 82, 1532, 1977a.

Baker, D.N., and E.C. Stone, The magnetopause electron layer along the distant magnetotail, Geophys. Res. Letters, 4, 133, $1977 \mathrm{~b}$.

Behannon, K.W., Mapping of the earth's bow shock and magnetic tail by Explorer 33, J. Geophys. Res., 73, 907, 1968.

Domingo, V., D.E. Page, and K. -P. Wenzel, Energetic electrons at the magnetopause, in Correlated Interplanetary and Magnetospheric observations, edited by D.E. Page, p. 159, D. Reidel, Dordrecht, The Netherlands, 1974.

Domingo, V., D.E. Page, and K.-P. Wenze1, Energetic and relativistic electrons near the polar magnetopause, J. Geophys. Res., 82, 2327, 1977.

Heikkfla, W.J., Is there an electrostatic field tangential to the dayside magnetopause and neutra1 line?, Geophys. Res. Letters, 2, 154, 1975.

Lampe, M. and K. Papadopoulos, Formation of fast electron talls in type II solar bursts, Astrophys. J., 212, 886, 1977.

Meng, C. -I., and K.A. Anderson, A layer of energetic electrons ( $>40 \mathrm{keV}$ ) near the magnetopause, J. Geophys. Res., 75, 1827, 1970.

Meng, C.-I., and K.A. Anderson, Characteristics of the magnetopause energetic electron layer; J. Geophys. Res., 80, 4237, 1975.

Reiff, P.H., and D.L. Reasoner, The magnetosheath electron population at lunar distance: General features, J. Geophys. Res., 80, 1232, 1975.

(Received July 29, 1977; accepted August 11, 1977) 